SECTION 7. Mechanics and machine construction.

Serhii Aleksandrovych Kraichuk

Applicant,

Institute of Hydromechanics of National Academy of Sciences of Ukraine

akraychuk@pisem.net

Natalia Serhiivna Remez

Professor, Doctor of Technical Sciences, National Technical University of Ukraine "Kyiv Polytechnic Institute"

NATALY.REMEZ@,gmail.com

Oksana Yaroslavivna Tverda

$\mathrm{PhD}$, Senior Lecturer

National Technical University of Ukraine "Kyiv Polytechnic Institute"

tverdaya@ukr.net

Serhii Vasyliovych Dyniak

Applicant

National Technical University of Ukraine "Kyiv Polytechnic Institute"

kkttkk297@gmail.com

\title{
METHOD OF PREDICTING THE SEISMIC-RESISTANT OF PROTECTED OBJECTS NEAR BLASTING
}

Abstract: The method of numerical calculation of the parameters of seismic waves by the explosion of cylindrical charges group in soil array and their interaction with the soil foundation of protected structures, which allows on the basis of complex applications calculate seismic load on the subject taking into account its geometrical and inertial characteristics and parameters of blasting and ground conditions is developed.

Key words: explosion, seismic wave, the protected object, mathematical modeling.

Citation: Kraichuk SA, Remez NS, Tverda OYa, Dyniak SV (2014) METHOD OF PREDICTING THE SEISMIC-RESISTANT OF PROTECTED OBJECTS NEAR BLASTING. ISJ Theoretical \& Applied Science 9 (17): 68-73. doi: http://dx.doi.org/10.15863/TAS.2014.09.17.11

\section{УДК 622.235}

\section{МЕТОДИКА ПРОГНОЗИРОВАНИЯ СЕЙСМОСТОЙКОСТИ ОХРАНЯЕМЫХ ОБЬЕКТОВ ВБЛИЗИ ПРОВЕДЕНИЯ ВЗРЫВНЫХ РАБОТ}

Аннотация: Разработана методика численного расчета параметров сейсмовзрывных волн при взрыве группь цилиндрических зарядов в грунтовом массиве и их взаимодействии с грунтовым основанием охраняемых сооружений, которая позволяет на основе созданного комплекса прикладных программ рассчитывать сейсмическую нагрузку на объект с учетом его геометрических и инеричинных характеристик, параметров взрывных работ и грунтовых условий.

Ключевые слова: взрыв, сейсмическая волна, охраняемый объект, математическое моделирование.

\section{ВСТУПЛЕНИЕ}

Использование энергии взрыва зарядов химических взрывчатых веществ, расширение объемов взрывных работ и масштабов взрывов обуславливают необходимость 
четкого расчета и создания эффективных методов управления воздействиями сейсмических волн. При этом возникает проблема прогнозируемой оценки устойчивых свойств сооружений, коммуникаций, расположенных вблизи карьерного поля, решение которой невозможно без точного знания влияния и характера действия внешних сил.

Существующие методики оценки сейсмической безопасности при проведении взрывных работ имеют ряд недостатков. Экспериментальные методики затратные и эффективны лишь для конкретных условий. Наиболее часто применяемые расчетные методики базируются на формуле Садовского уточненной различными коэффициентами или привязкой их к паспортным физико-механическим константам почв. Еще одним недостатком расчетных методик является то, что упомянутая формула, основанная на принципе геометрического и энергетического сходства справедлива для взрыва единичного сосредоточенного заряда, что приводит к недостоверной количественной оценке сейсмобезопасных параметров проведения взрывных работ.

Хотя выбор параметров взрывных работ определяет как полезный эффект взрыва по измельчению горной породы, так и сейсмический эффект взрыва, до сих пор этот вопрос не достаточно исследован в связанной постановке. Поэтому разработка эффективной методики исследования параметров сейсмовзрывных волн (CBB), влияющих на сейсмическую устойчивость охраняемых объектов при взрывах группы цилиндрических зарядов с учетом условий проведения взрывных работ и амплитудно-временных и физикомеханических свойств грунтов и объектов является актуальной научной задачей.

Теоретические исследования механизма разрушения горных пород при взрывах групповых и одиночных зарядов в большинстве случаев проведены для зарядов сферической симметрии [1-4]. При этом используются упрощенные представления о развитии волновых процессов в массиве: породный массив считается идеально упругим, ударные волны отсутствуют. Теоретические решения получены в потенциальном приближении [5].

Исследование взрыва группы цилиндрических зарядов, которые чаще всего используются в практике ведения взрывных работ, часто базируется на замене удлиненного заряда цепочкой элементарных сосредоточенных зарядов $[1-2,6]$, или закономерности, полученные при решении одномерных задач, обобщаются для пространственных зависимостей [7]. В работах [8-9] поставлены и решены пространственные задачи о взрыве одного или двух цилиндрических зарядов в почвенном пространстве, однако результаты этих решений в полной мере не раскрывают механизм суперпозиции.

Большинство работ по исследованию устойчивости сооружений посвящено изучению влияния сейсмических волн от землетрясений [1-5]. Исследований влияния сейсмовзрывных волн на природные и инженерные объекты значительно меньше [6-7]. Исходя из проведенного анализа научных и практических достижений и выявленных при этом недостатков по данному направлению, сформулирована цель исследования.

Целью работы является разработка методики расчета динамических процессов при распространении волн от одиночных и групповых взрывов цилиндрических зарядов в грунтовом массиве и их взаимодействии с охраняемыми объектами, для усовершенствования способа прогнозирования их сейсмостойкости.

\section{РЕЗУЛЬТАТЫ ИССЛЕДОВАНИЙ}

На базе алгоритмов расчетов проведенных в предыдущих разделах была разработана методика определения сейсмобезопасных параметров взрывных работ, основанная на математическом моделировании закономерностей распространения сейсмовзрывных волн в грунтовом массиве и их взаимодействия с охраняемым объектом.

На основе разработанной методики был реализован пакет прикладных программ на языке программирования DELFI для оперативного расчета сейсмобезопасных параметров взрывных работ, который предлагает для этого решение трех взаимосвязанных и 
последовательных задач (выбор задачи можно осуществить в главном окне программы, или просто следовать последовательности задач):

- расчет параметров сейсмовзрывной волны при взрыве цилиндрического заряда в грунтовом массиве или горной породе;

- расчет параметров сейсмовзрывной волны при взрыве группы цилиндрических зарядов при мгновенном или короткозамедленном взрывании;

- расчет амплитудно-частотного взаимодействия основы охраняемого объекта и падающей сейсмической волны.

Общий алгоритм методики приведен на рисунке 1, на котором изображена блоксхема последовательности расчетов. Блок-схема состоит из блока входных данных, трех блоков постановки расчетов, блока передачи полученных результатов и блока принятия решения о повторении или завершении расчета на основе сравнения результатов с нормативными значениями скорости колебаний для охраняемых объектов.

Алгоритм состоит из четырех этапов. На первом этапе вычисляются параметры волновых процессов для взрыва одного цилиндрического заряда взрывчатого вещества в грунтовом массиве, что взрывается мгновенно на определенном расстоянии от свободной поверхности. Математическая постановка задачи приведена под №1 в виде системы квазилинейных дифференциальных уравнений в частных производных, описывающей движение почвы и продуктов детонации (ПД), с соответствующими начальными и граничными условиями. Входными данными являются значения физико-механических характеристик горной породы в месте проведения взрыва, детонационные характеристики взрывчатых веществ, параметры конструкции зарядов. Для решения этой задачи применялся метод конечных разностей с использованием явной разностной схемы типа «крест».

В результате численного эксперимента получаем массив основных параметров СВВ в зависимости от времени и относительного расстояния (рисунок 2). При обработке полученных данных методом наименьших квадратов получаем аналитические зависимости напряжений, скоростей и характерных времен взрыва (времени прихода импульса в данную точку массива, достижения максимума, окончания действия импульса), которые являются исходными данными для расчета суперпозиции волн при различных условиях взрыва.

На втором этапе проводятся аналитические расчеты полей напряжений и скоростей для группового взрыва в зависимости от условий взрывания: глубины залегания, количества зарядов, расстояния между ними, наличия или отсутствия КЗВ (задача №2 на рисунке 1). Входными параметрами являются количество зарядов $n$, расстояние между ними $a$, расстояние до свободной поверхности $h$, время замедления $t_{o ̈ i}$ и относительное расстояние до места наблюдения $\bar{r}$. Предусмотрена возможность одинакового времени замедления для всех зарядов или введения времени замедления отдельно для каждого заряда. Также задается момент времени для которого рассчитываются поля напряжений и скоростей и размеры расчетной сетки (м), покрывающей область для которой производится расчет. На рисунке 2 приведено окно вывода результатов для этой же задачи. Результаты расчета выводятся отдельным окном с четырьмя вкладками - по две проекции напряжений и скоростей по осям $X$ и $Y$. Также выводятся максимальные значения всех рассчитанных величин в верхней части окна. Поля величин автоматически изображаются в виде разноцветных областей ограниченных изолиниями, их цвет и количество можно задать в главном окне, во вкладке меню «Конфигурация». Предусмотрена возможность изменения количества областей и значений изолиний, которые ограничивают, в правой части окна вывода результатов второй задачи. 


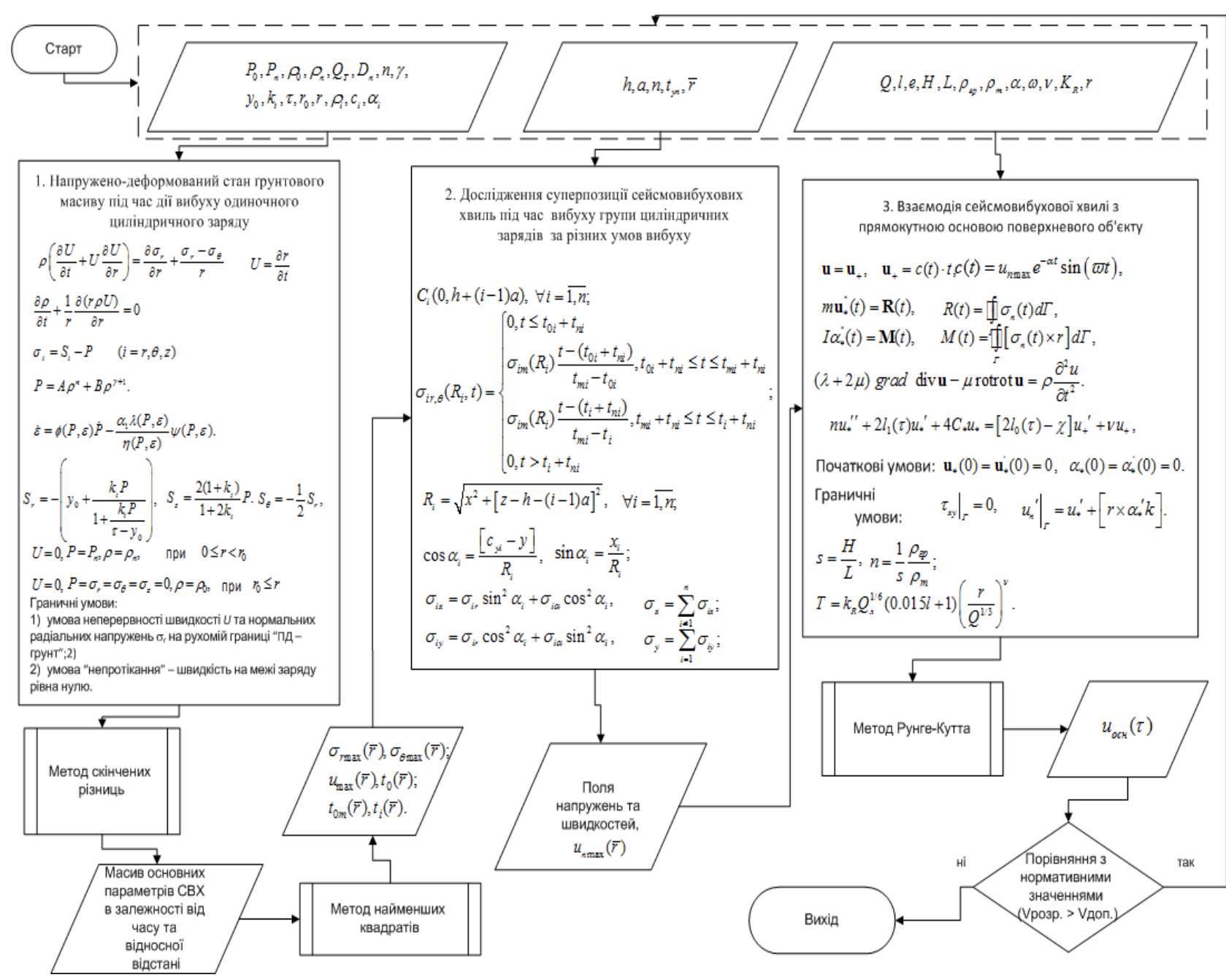

Рисунок 1 - Блок-схема последовательности расчетов.

На основе полученных зависимостей максимальных скоростей от времени на третьем этапе проводится расчет взаимодействия СВВ с основой охраняемого объекта с учетом линейных размеров объекта, его инерционных характеристик, амплитудночастотных характеристик падающей волны. Результатами расчета являются графические зависимости перемещения и скорости колебаний основы охраняемого объекта от времени (задача 3).

На результирующих графиках определяются максимальные амплитуды скоростей колебания основы объекта и сравниваются с приведенными в окне программы допустимыми нормативными значениями скоростей колебаний грунта в основании охраняемых объектов. Окна ввода данных и вывода результатов задачи о взаимодействии сейсмической волны с основанием охраняемого объекта изображены на рисунке 3.

В зависимости от величины допустимой скорости колебаний определенной нормативами для данного охраняемого объекта, на основе полученных результатов можно дать рекомендации о необходимости изменения начальных параметров взрывных работ с целью снижения сейсмического эффекта взрыва и повторить расчет сейсмостойкости объекта для новых параметров взрывных работ. При этом можно варьировать параметрами заряда, типом ВВ, условиями взрывания. Алгоритм расчета сейсмобезопасных параметров взрыва повторяется до тех пор, пока не будут достигнуты оптимальные параметры ведения взрывных работ, обеспечивающих эффективное разрушение грунтового массива и сейсмическую безопасность охраняемого объекта. 


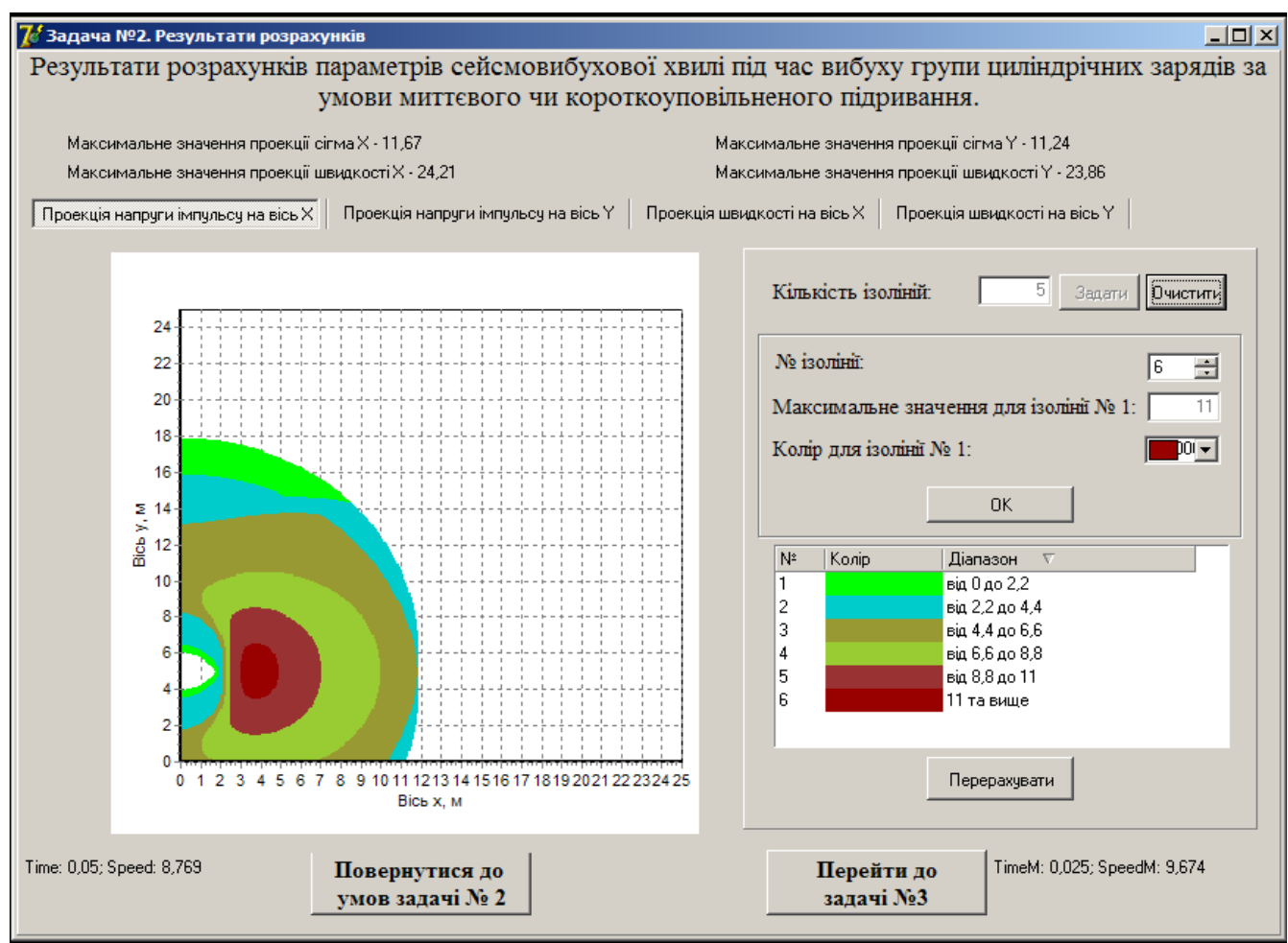

Рисунок 2 - Окно введения данных и вывода результатов задачи о взрыве группы цилиндрических зарядов в грунтовом массиве.

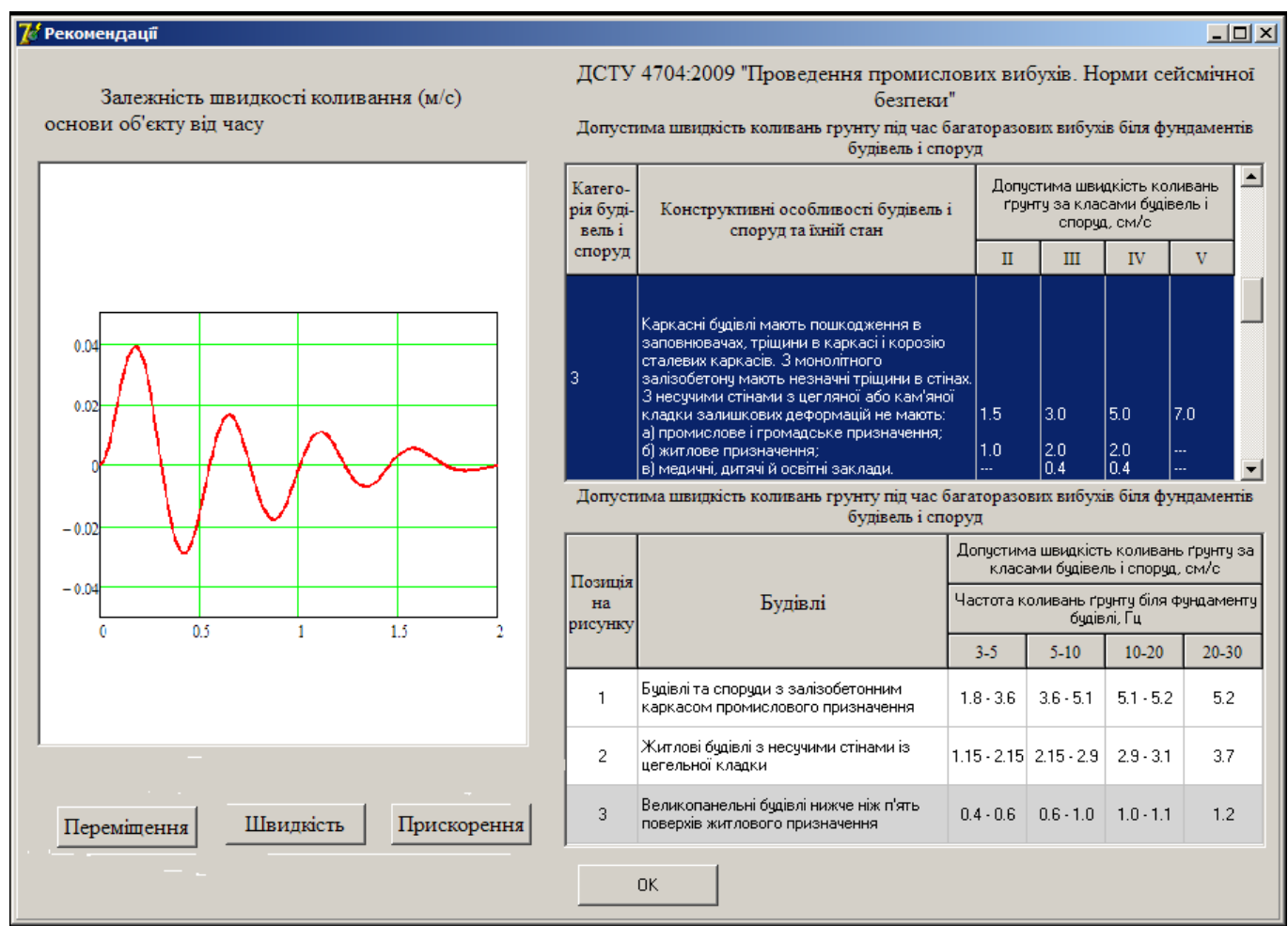

Рисунок 3 - Окно введения данных и вывода результатов задачи о взаимодействии сейсмической волны с основанием охраняемого объекта.

Следует отметить, что разработанная методика численного эксперимента по установлению сейсмобезопасных параметров взрывных работ вблизи охраняемых объектов имеет ряд преимуществ по сравнению с натурным экспериментом. Во-первых, она менее ресурсоемкая и дорого стоимостная. 
Во-вторых, она позволяет получить результаты для различных начальных условий достаточно оперативно. К недостаткам работы следует отнести необходимость подтверждения теоретическими данными экспериментальных исследований.

\section{ВЫВОДЫ}

1 Разработана методика прогнозирования сейсмостойкости охраняемых объектов при взрывах группы цилиндрических зарядов, которые на основе исходных параметров взрывных работ, грунтовых условий и характеристик объекта позволяют рассчитать сейсмическую нагрузку на объект и в случае превышения допустимых норм скорректировать параметры взрыва

2 Разработан алгоритм и компьютерная программа расчета взаимодействия грунтового основания с сооружением под действием СВВ взрыва группы цилиндрических зарядов с учетом детонационных свойств ВВ, физико-механических характеристик грунтов и условий взрывания, позволяющие рассчитать амплитудно-частотные характеристики охраняемого объекта

3 Разработан прикладной комплекс программ, для численного расчета параметров СВВ при взрыве одного и группы цилиндрических зарядов в грунтовом массиве, и амплитудно-частотных параметров падающей сейсмической волны с учетом взаимодействия грунтового основания и охраняемого объекта, который позволяет на основе исходных параметров взрывных работ, детонационных характеристик ВВ, грунтовых условий и геометрических и инерционных характеристик объекта рассчитать сейсмическую нагрузку на объект и в случае необходимости скорректировать параметры взрыва.

\section{References:}

1. Shemyakin EI (1963) O volnakh napryazheniy v prochnykh gornykh porodakh. PMTF, pp.83-93.

2. Borovikov VA, Vanyagin VA (1976) K raschetu parametrov volny napryazheniya pri vzryve udlinennogo zaryada v gornykh porodakh. Vzryv. delo, No. 76/33, pp. 74-85.

3. Kravets VG (1979) Dinamika uplotneniya gruntovogo massiva vzryvom. Kiev: Nauk. dumka, 134.

4. Rodionov VN (1971) Mekhanicheskiy effekt podzemnogo vzryva. Moscow, Nedra, 200.

5. Mikhalyuk AV (1980) Gornye porody pri neravnomernykh dinamicheskikh nagruzkakh. Kiev: Nauk. dumka, 154.

6. Bazhenov VG, Kochetkov AV, Fel'dgun VR (1989) Deformirovanie tsilindricheskoy obolochki $\mathrm{V}$ myagkoy gruntovoy srede pod deystviem vnutrennego impul'snogo nagruzheniya. Prikladnye problemy prochnosti i plastichnosti. Metody resheniya: Vsesoyuz. mezhvuz. sb., pp. 87-95.

7. Komir VM (1979) Osnovy teorii i metody vzryvnogo drobleniya gornykh porod. Kiev: Nauk. dumka, 224.

8. Frolov OO (2006) Osoblivosti rozrakhunku ob'emu ruynuvannya skel'nikh porid pid chas vibukhu sverdlovinnogo zaryadu z urakhuvannyam khvil' napruzhen'. Visnik NTTU «KPI». Seriya «Girnitstvo»: Zb. nauk. prats, Vip. 14, pp. 93-101.

9. Frolov OO (2008) Teoretichni peredumovi rozrakhunku ob'emu ruynuvannya girs'kikh porid pid chas vibukhu sistem podovzhenikh zaryadiv. Visnik NTTU «KPI». Seriya «Girnitstvo»: Zb. nauk. prats, Vip. 16, pp. 13-16. 